\title{
HARDY AND HARDY-RELLICH TYPE INEQUALITIES WITH BESSEL PAIRS
}

\author{
Nguyen Lam \\ University of British Columbia, Department of Mathematics \\ and The Pacific Institute for the Mathematical Sciences \\ Vancouver, BC V6T1Z4, Canada; nlam@math.ubc.ca
}

\begin{abstract}
Using the Bessel pairs in the sense of Ghoussoub and Moradifam [16], we provide the necessary and sufficient conditions on a pair of positive functions so that the Hardy type inequalities in the spirit of Badiale-Tarantello [3] hold. We also set up the requirements for a pair of potentials so that the Hardy-Rellich type inequalities with radial derivatives are valid.
\end{abstract}

\section{Introduction}

We first recall the celebrated Hardy inequality in $\mathbf{R}^{N}, N \geq 3$ : Let $u \in D^{1,2}\left(\mathbf{R}^{N}\right)$ with $N \geq 3$. Then $\frac{u^{2}}{|x|^{2}} \in L^{1}\left(\mathbf{R}^{N}\right)$. Moreover,

$$
\left(\frac{N-2}{2}\right)^{2} \int_{\mathbf{R}^{N}} \frac{u^{2}}{|x|^{2}} d x \leq \int_{\mathbf{R}^{N}}|\nabla u|^{2} d x
$$

and $\left(\frac{N-2}{2}\right)^{2}$ is the best possible constant.

It is well-known that the optimal constant $\left(\frac{N-2}{2}\right)^{2}$ is never achieved. Hence, we may ask for the improved versions of the Hardy inequalities where extra nonnegative terms can be added to the LHS of (1.1). On the whole space $\mathbf{R}^{N}$, Ghoussoub and Moradifam showed in [18] that there is no strictly positive $V \in V^{1}((0, \infty))$ such that the inequality

$$
\int_{\mathbf{R}^{N}}|\nabla u|^{2} d x-\left(\frac{N-2}{2}\right)^{2} \int_{\mathbf{R}^{N}} \frac{u^{2}}{|x|^{2}} d x \geq \int_{\mathbf{R}^{N}} V(|x|) u^{2} d x
$$

holds for all $u \in C_{0}^{\infty}\left(\mathbf{R}^{N}\right)$. However, the situation on bounded domain is different. Indeed, let $\Omega$ be a bounded domain in $\mathbf{R}^{N}, N \geq 3$, with $0 \in \Omega$. Then Brezis and Vázquez proved in [8] that for all $u \in W_{0}^{1,2}(\Omega)$ :

$$
\int_{\Omega}|\nabla u|^{2} d x-\left(\frac{N-2}{2}\right)^{2} \int_{\Omega} \frac{u^{2}}{|x|^{2}} d x \geq z_{0}^{2} \omega_{N}^{\frac{2}{N}}|\Omega|^{-\frac{2}{N}} \int_{\Omega} u^{2} d x
$$

where $\omega_{N}$ is the volume of the unit ball and $z_{0}=2.4048 \ldots$ is the first zero of the Bessel function $J_{0}(z)$. The constant $z_{0}^{2} \omega_{N}^{\frac{2}{N}}|\Omega|^{-\frac{2}{N}}$ is optimal when $\Omega$ is a ball. However, $z_{0}^{2} \omega_{N}^{\frac{2}{N}}|\Omega|^{-\frac{2}{N}}$ is not attained in $W_{0}^{1,2}(\Omega)$. Hence, it is natural to conjecture that $z_{0}^{2} \omega_{N}^{\frac{2}{N}}|\Omega|^{-\frac{2}{N}} \int_{\Omega} u^{2} d x$ is just a first term of an infinite series of extra terms that can be added to the RHS of (1.2). This problem was investigated by many authors. See

https://doi.org/10.5186/aasfm.2018.4308

2010 Mathematics Subject Classification: Primary 26D10, 35A23, 46E35.

Key words: Bessel pairs, Hardy inequalities, Hardy-Rellich inequalities, Heisenberg-PauliWeyl uncertainly principle, best constants.

Research of this work was partially supported by the PIMS-Math Distinguished Post-doctoral Fellowship from the Pacific Institute for the Mathematical Sciences. 
$[1,6,9,10,13,14,15,20,21,22,23,27,28,36]$, to name just a few. See also the books $[24,31]$ that are by now standard references on Hardy inequalities.

In [16, 17], Ghoussoub and Moradifam proved the following result to improve, extend and unify several results about the Hardy type inequalities:

Theorem A. Let $0<R \leq \infty, V$ and $W$ be positive radial $C^{1}$-functions on $B_{R} \backslash\{0\}$ such that $\int_{0}^{R} \frac{1}{r^{N-1} V(r)} d r=\infty$ and $\int_{0}^{R} r^{N-1} V(r) d r<\infty$. Then the following are equivalent:

(1) $(V, W)$ is a $N$-dimensional Bessel pair on $(0, R)$.

(2) $\int_{B_{R}} V(x)|\nabla u|^{2} d x \geq \beta(V, W ; R) \int_{B_{R}} W(x)|u|^{2} d x$ for all $u \in C_{0}^{\infty}\left(B_{R}\right)$ with $\beta(V, W ; R)$ being the best constant.

Here we say that a couple of $C^{1}$-functions $(V, W)$ is a $N$-dimensional Bessel pair on $(0, R)$ if there exists $c>0$ such that the ordinary differential equation

$$
y^{\prime \prime}(r)+\left(\frac{N-1}{r}+\frac{V_{r}(r)}{V(r)}\right) y^{\prime}(r)+\frac{c W(r)}{V(r)} y(r)=0
$$

has a positive solution on the interval $(0, R)$. Also, $\beta(V, W ; R)$ is defined as the supremum of such $c$. It can be also verified that $(V, W)$ is a $N$-dimensional Bessel pair on $(0, R)$ if and only if $\left(r^{N-1} V, r^{N-1} W\right)$ is a 1-dimensional Bessel pair on $(0, R)$. See the book [17] for more properties and examples about the $N$-dimensional Bessel pair.

It has been observed that we can improve the Hardy inequalities using the radial derivative as follows: Using the ideas in [28], for $\varepsilon>0$, we denote

$$
U_{\varepsilon}(x)=\left(\frac{x_{1}}{\varepsilon+|x|^{2}} u^{2}, \ldots, \frac{x_{N}}{\varepsilon+|x|^{2}} u^{2}\right) .
$$

Applying the divergence theorem for the smooth vector field $U_{\varepsilon}$, we obtain

$$
\begin{aligned}
\int_{\mathbf{R}^{N}} \frac{N \varepsilon+(N-2)|x|^{2}}{\left(\varepsilon+|x|^{2}\right)^{2}} u^{2}(x) d x & =-2 \int_{\mathbf{R}^{N}} \frac{(x \cdot \nabla u) u(x)}{\varepsilon+|x|^{2}} d x \\
& \leq 2 \int_{\mathbf{R}^{N}} \frac{|x||u(x)|}{\varepsilon+|x|^{2}}\left|\frac{x}{|x|} \cdot \nabla u\right| d x \\
& \leq 2\left(\int_{\mathbf{R}^{N}}\left(\frac{|x||u(x)|}{\varepsilon+|x|^{2}}\right)^{2} d x\right)^{\frac{1}{2}}\left(\int_{\mathbf{R}^{N}}\left|\frac{x}{|x|} \cdot \nabla u\right|^{2} d x\right)^{\frac{1}{2}} .
\end{aligned}
$$

Hence, letting $\varepsilon \downarrow 0$, we obtain

$$
\left(\frac{N-2}{2}\right)^{2} \int_{\mathbf{R}^{N}} \frac{u^{2}}{|x|^{2}} d x \leq \int_{\mathbf{R}^{N}}\left|\frac{x}{|x|} \cdot \nabla u\right|^{2} d x \leq \int_{\mathbf{R}^{N}}|\nabla u|^{2} d x .
$$

Of course, the constant $\left(\frac{N-2}{2}\right)^{2}$ is still optimal.

It can be noted that $\frac{x}{|x|} \cdot \nabla u$ is actually the radial gradient of $u$. Indeed, in the polar coordinate, $\left|\frac{x}{|x|} \cdot \nabla u\right|=\left|\partial_{r} u(r \sigma)\right|$ while $|\nabla u|=\left(\left|\partial_{r} u(r \sigma)\right|^{2}+\frac{\left|\nabla_{\mathbf{S}^{N-1}} u(r \sigma)\right|^{2}}{r^{2}}\right)^{\frac{1}{2}}$. Obviously, (1.3) is (1.1) when $u$ is radial. We would also like to mention here the role of the operator $\mathcal{R}=\frac{x}{|x|} \cdot \nabla$ in the literature. One of the interesting problems is to investigate the Hardy and Hardy-Rellich inequalities on general homogeneous groups. However, as explained in [34], since these spaces do not have to be stratified 
or even graded, the concept of horizontal gradients do not make sense. Thus, it is logical to work with the full gradient. On the other hand, unless the homogeneous groups are abelian, the full gradient is not homogeneous. Nevertheless, on the homogeneous groups, we can define the operator $\mathcal{R}$ that is homogeneous of order -1 and is analogous to the usual Euclidean gradient and to the radial derivative $\frac{x}{|x|} \cdot \nabla$ on $\mathbf{R}^{n}$. Hence, it is important and interesting to investigate the functional and geometric inequalities under the radial derivative operator $\mathcal{R}=\frac{x}{|x|} \cdot \nabla$. Actually, the Hardy type inequalities with radial gradient have been intensively studied recently. See [20, 21, 27, 32, 36], for example.

Motivated by the results in $[16,17]$ and the above observation, it was proved in $[25,26]$ the following result:

Theorem B. Let $0<R \leq \infty, V$ and $W$ be positive radial functions on $B_{R} \backslash\{0\}$. Then the following are equivalent:
(A) $\int_{B_{R}} V(x)|\nabla u|^{2} d x \geq \int_{B_{R}} W(x)|u|^{2} d x$ for all $u \in C_{0}^{\infty}\left(B_{R}\right)$.
(B) $\int_{B_{R}} V(x)|\nabla u|^{2} d x \geq \int_{B_{R}} W(x)|u|^{2} d x$ for all radial functions $u \in C_{0}^{\infty}\left(B_{R}\right)$.
(C) $\int_{B_{R}} V(x)\left|\frac{x}{|x|} \cdot \nabla u\right|^{2} d x \geq \int_{B_{R}} W(x)|u|^{2} d x$ for all $u \in C_{0}^{\infty}\left(B_{R}\right)$.

It is worth mentioning here that in statement (B) of Theorem B, the Hardy inequality just holds for radial functions. Noting that since $V$ and $W$ are just positive radial functions, we can not apply the classical Schwarz rearrangement arguments to deduce (A) in general. In [26], to show $(\mathrm{B}) \Rightarrow(\mathrm{C}) \Rightarrow(\mathrm{A}) \Rightarrow(\mathrm{B})$, we use the ideas in $[16,37]$ to decompose the functions $u$ in $C_{0}^{\infty}\left(B_{R}\right)$ into spherical harmonics and then will only work with radial components.

In [3], instigated by a nonlinear elliptic equation arising in astrophysics, Badiale and Tarantello investigated the following Hardy type inequalities:

$$
\int_{\mathbf{R}^{N}} \frac{|u(x)|^{p}}{|y|^{p}} d x \leq C_{N, k, p} \int_{\mathbf{R}^{N}}|\nabla u(x)|^{p} d x
$$

where $x=(y, z) \in \mathbf{R}^{k} \times \mathbf{R}^{N-k}$. The optimal constant $C_{N, k, p}=\left(\frac{p}{k-p}\right)^{p}$ was also conjectured in [3] and then verified in [35]. Stimulated by this result and the developments in $[16,17,25,26]$, we will prove in this note the following result:

Theorem 1.1. Let $V$ and $W$ be positive radial $C^{1}$-functions on $\mathbf{R}^{k} \backslash\{0\}$ such that $\int_{0}^{\infty} \frac{1}{r^{k-1} V(r)} d r=\infty$ and $\int_{0}^{\infty} r^{k-1} V(r) d r<\infty$. Then the following are equivalent:

(1) $\left(r^{k-1} V, r^{k-1} W\right)$ is a 1-dimensional Bessel pair on $(0, \infty)$.

(2) $\int_{\mathbf{R}^{N-k}} \int_{\mathbf{R}^{k}} V(|y|)\left|\frac{y}{|y|} \cdot \nabla_{y} u(y, z)\right|^{2} d y d z \geq c \int_{\mathbf{R}^{N-k}} \int_{\mathbf{R}^{k}} W(|y|)|u(y, z)|^{2} d y d z$ for all $u \in C_{0}^{\infty}\left(\mathbf{R}^{N}\right)$ for some $c>0$.

(3) $\int_{\mathbf{R}^{N-k}} \int_{\mathbf{R}^{k}} V(|y|)\left|\nabla_{y} u(y, z)\right|^{2} d y d z \geq c \int_{\mathbf{R}^{N-k}} \int_{\mathbf{R}^{k}} W(|y|)|u(y, z)|^{2} d y d z$ for all $u \in C_{0}^{\infty}\left(\mathbf{R}^{N}\right)$ for some $c>0$.

(4) $\int_{\mathbf{R}^{N-k}} \int_{\mathbf{R}^{k}} V(|y|)|\nabla u(y, z)|^{2} d y d z \geq c \int_{\mathbf{R}^{N-k}} \int_{\mathbf{R}^{k}} W(|y|)|u(y, z)|^{2} d y d z$ for all $u \in C_{0}^{\infty}\left(\mathbf{R}^{N}\right)$ for some $c>0$.

Moreover, $\beta\left(r^{k-1} V, r^{k-1} W ; \infty\right)$ is the optimal constant. 
As a consequence of Theorem 1.1, we can get the following version of the Heisenberg-Pauli-Weyl uncertainly principle: Let $V$ and $W$ be positive radial $C^{1}$-functions on $\mathbf{R}^{k} \backslash\{0\}$ such that $\int_{0}^{\infty} \frac{1}{r^{k-1} V(r)} d r=\infty, \int_{0}^{\infty} r^{k-1} V(r) d r<\infty$ and $\left(r^{k-1} V, r^{k-1} W\right)$ is a 1-dimensional Bessel pair on $(0, \infty)$. Then we have:

$$
\begin{aligned}
& \beta\left(r^{k-1} V, r^{k-1} W ; \infty\right)\left(\int_{\mathbf{R}^{N-k}} \int_{\mathbf{R}^{k}}|u(y, z)|^{2} d y d z\right)^{2} \\
& =\beta\left(r^{k-1} V, r^{k-1} W ; \infty\right)\left(\int_{\mathbf{R}^{N-k}} \int_{\mathbf{R}^{k}} \sqrt{W(|y|) \mid}|u(y, z)| \frac{|u(y, z)|}{\sqrt{W(|y|)}} d y d z\right)^{2} \\
& \leq\left(\int_{\mathbf{R}^{N-k}} \int_{\mathbf{R}^{k}} W(|y|)|u(y, z)|^{2} d y d z\right)\left(\int_{\mathbf{R}^{N-k}} \int_{\mathbf{R}^{k}} \frac{|u(y, z)|^{2}}{W(|y|)} d y d z\right) \\
& \leq\left(\int_{\mathbf{R}^{N-k}} \int_{\mathbf{R}^{k}} V(|y|)\left|\frac{y}{|y|} \cdot \nabla_{y} u(y, z)\right|^{2} d y d z\right)\left(\int_{\mathbf{R}^{N-k}} \int_{\mathbf{R}^{k}} \frac{|u(y, z)|^{2}}{W(|y|)} d y d z\right) .
\end{aligned}
$$

An example is the 1-dimensional Bessel pair $\left(r^{k-1}, r^{k-1} r^{-2}\right)$ on $(0, \infty)$. In this case, $\beta\left(r^{k-1}, r^{k-1} r^{-2} ; \infty\right)=\left(\frac{k-2}{2}\right)^{2}$ and thus,

$$
\begin{aligned}
& \left(\int_{\mathbf{R}^{N-k}} \int_{\mathbf{R}^{k}}|\nabla u(y, z)|^{2} d y d z\right)\left(\int_{\mathbf{R}^{N-k}} \int_{\mathbf{R}^{k}}|y|^{2}|u(y, z)|^{2} d y d z\right) \\
& \geq\left(\int_{\mathbf{R}^{N-k}} \int_{\mathbf{R}^{k}}\left|\frac{y}{|y|} \cdot \nabla_{y} u(y, z)\right|^{2} d y d z\right)\left(\int_{\mathbf{R}^{N-k}} \int_{\mathbf{R}^{k}}|y|^{2}|u(y, z)|^{2} d y d z\right) \\
& \geq\left(\frac{k-2}{2}\right)^{2}\left(\int_{\mathbf{R}^{N-k}} \int_{\mathbf{R}^{k}}|u(y, z)|^{2} d y d z\right)^{2} .
\end{aligned}
$$

Especially, when $k=N$, we recover the well-known Heisenberg-Pauli-Weyl uncertainly principle which is one of the most famous problems in mathematical physics and classical Fourier analysis alike:

$$
\frac{N-2}{2} \int_{\mathbf{R}^{N}} u^{2} d x \leq\left(\int_{\mathbf{R}^{N}}|x|^{2} u^{2} d x\right)^{\frac{1}{2}}\left(\int_{\mathbf{R}^{N}}|\nabla u|^{2} d x\right)^{\frac{1}{2}} .
$$

We also mention the paper [33] where the Heisenberg-Pauli-Weyl uncertainly principle on homogeneous groups is discussed.

Along the same line of thought, we also get the following characterization in the spirit of $[16,18,19]$ :

Theorem 1.2. Let $p>1, V$ and $W$ be positive radial $C^{1}$-functions on $\mathbf{R}^{k} \backslash\{0\}$. Assume that there is a positive function $\phi \in C^{2}(0, \infty)$ such that

$$
\left(r^{k-1} V(r)\left|\phi^{\prime}\right|^{p-2} \phi^{\prime}\right)^{\prime}+r^{k-1} W(r) \phi^{p-1} \leq 0 \quad \text { on }(0, \infty)
$$

and

Then we have

$$
\lim _{r \downarrow 0} r^{k-1} V(r) \phi(r)\left|\phi_{r}(r)\right|^{p-2} \phi_{r}(r)\left|\frac{u(r)}{\phi(r)}\right|^{p}=0 .
$$

$$
\int_{\mathbf{R}^{N-k}} \int_{\mathbf{R}^{k}} V(|y|)\left|\frac{y}{|y|} \cdot \nabla_{y} u(y, z)\right|^{p} d y d z \geq \int_{\mathbf{R}^{N-k}} \int_{\mathbf{R}^{k}} W(|y|)|u(y, z)|^{p} d y d z
$$

for all $u \in C_{0}^{\infty}\left(\mathbf{R}^{N}\right)$. 
We now concern the well-known Hardy-Rellich inequality: for $u \in C_{0}^{\infty}\left(\mathbf{R}^{N} \backslash\{0\}\right)$, $N \geq 5$ :

$$
\left(\frac{N(N-4)}{4}\right)^{2} \int_{\mathbf{R}^{N}} \frac{u^{2}}{|x|^{4}} d x \leq \int_{\mathbf{R}^{N}}|\Delta u|^{2} d x .
$$

Many research papers have tried to investigate the improved Hardy-Rellich inequalities of the form

$$
\int_{\mathbf{R}^{N}}|\Delta u|^{2} d x-\left(\frac{N(N-4)}{4}\right)^{2} \int_{\mathbf{R}^{N}} \frac{u^{2}}{|x|^{4}} d x \geq \int_{\mathbf{R}^{N}} W(|x|) u^{2} d x
$$

where $W$ is certain explicit radially symmetric potential of order lower than $\frac{1}{|x|^{4}}$. For examples, see $[2,5,7,11,12,29,30,34]$. Again, in [16, 17], the authors have proved the following general result to complete, simplify and improve many known results:

Theorem C. Let $0<R \leq \infty, V$ and $W$ be positive radial $C^{1}$-functions on $B_{R} \backslash\{0\}$ such that $\int_{0}^{R} \frac{1}{r^{N-1} V(r)} d r=\infty$ and $\int_{0}^{R} r^{N-1} V(r) d r<\infty$. Then the following are equivalent:

(1) $(V, W)$ is a $N$-dimensional Bessel pair on $(0, R)$.

(2) $\int_{B_{R}} V(x)|\Delta u|^{2} d x \geq c \int_{B_{R}} W(x)|\nabla u|^{2} d x+(N-1) \int_{B_{R}}\left(\frac{V(x)}{|x|^{2}}-\frac{V_{r}(x)}{|x|}\right)|\nabla u|^{2} d x$ for all radial functions $u \in C_{0}^{\infty}\left(B_{R}\right)$ for some $c>0$ with $\beta(V, W ; R)$ being the best constant.

In addition, if $W(x)-2 \frac{V(x)}{|x|^{2}}+2 \frac{V_{r}(x)}{|x|}-V_{r r}(x) \geq 0$ on $(0, R)$, then the above are equivalent to

$$
\begin{aligned}
& \int_{B_{R}} V(x)|\Delta u|^{2} d x \\
& \geq \beta(V, W ; R) \int_{B_{R}} W(x)|\nabla u|^{2} d x+(N-1) \int_{B_{R}}\left(\frac{V(x)}{|x|^{2}}-\frac{V_{r}(x)}{|x|}\right)|\nabla u|^{2} d x
\end{aligned}
$$

for all $u \in C_{0}^{\infty}\left(B_{R}\right)$ with $\beta(V, W ; R)$ being the best constant.

Our next motivation is as follows: There is no analog of homogeneous Laplacian or sub-Laplacian on general homogeneous groups. Moreover, there may be even no homogeneous hypoelliptic left-invariant differential operators. Indeed, the existence of such an operator would imply that the group must be graded. Hence, as above, it is natural to set up versions of the Hardy-Rellich inequalities using the radial derivatives $\mathcal{R}=\frac{x}{|x|} \cdot \nabla$. In fact, we will prove the following result:

Theorem 1.3. Let $0<R \leq \infty, V$ and $W$ be positive radial $C^{1}$-functions on $B_{R} \backslash\{0\}$ such that $\int_{0}^{R} \frac{1}{r^{N-1} V(r)} d r=\infty$ and $\int_{0}^{R} r^{N-1} V(r) d r<\infty$. Then the following are equivalent:

(1) $(V, W)$ is a $N$-dimensional Bessel pair on $(0, R)$.

(2) $\int_{B_{R}} V(|x|)\left|\mathcal{R}^{2} u+\frac{N-1}{|x|} \mathcal{R} u\right|^{2} d x$

$\geq c \int_{B_{R}} W(|x|)|\mathcal{R} u|^{2} d x+(N-1) \int_{B_{R}}\left(\frac{V(|x|)}{|x|^{2}}-\frac{V_{r}(|x|)}{|x|}\right)|\mathcal{R} u|^{2} d x$ for all $u \in$ $C_{0}^{\infty}\left(B_{R}\right)$ for some $c>0$ with $\beta(V, W ; R)$ being the best constant. 
For example, using Theorem 1.3 for a suitable explicit Bessel pair (see [16, 17]), we could obtain the following result:

$$
\int_{B_{R}}\left|\mathcal{R}^{2} u+\frac{N-1}{|x|} \mathcal{R} u\right|^{2} d x \geq \frac{N^{2}}{4} \int_{B_{R}} \frac{|\mathcal{R} u|^{2}}{|x|^{2}} d x+\frac{z_{0}^{2}}{R^{2}} \int_{B_{R}}|\mathcal{R} u|^{2} d x .
$$

This Hardy-Rellich type inequality seems new in the literature. Again, many examples and properties of Bessel pair were provided in [17]. Hence, from Theorem 1.3 we can get various versions of the Hardy-Rellich type inequalities with the operator $\mathcal{R}^{2}+\frac{N-1}{|x|} \mathcal{R}$. This also enables us to investigate the Hardy-Rellich type inequalities in the settings of homogeneous groups. See [34] for example.

\section{Proof of Theorem 1.1 and Theorem 1.2}

Proof of Theorem 1.1. First, assume that $\left(r^{k-1} V, r^{k-1} W\right)$ is a 1-dimensional Bessel pair on $(0, \infty)$. Fix $z \in \mathbf{R}^{N-k}$, by Theorem A and Theorem B, we get

$$
\int_{\mathbf{R}^{k}} V(|y|)\left|\frac{y}{|y|} \cdot \nabla_{y} u(y, z)\right|^{2} d y \geq \beta\left(r^{k-1} V, r^{k-1} W ; \infty\right) \int_{\mathbf{R}^{k}} W(|y|)|u(y, z)|^{2} d y .
$$

Hence

$$
\begin{aligned}
& \int_{\mathbf{R}^{N-k}} \int_{\mathbf{R}^{k}} V(|y|)|\nabla u(y, z)|^{2} d y d z \geq \int_{\mathbf{R}^{N-k}} \int_{\mathbf{R}^{k}} V(|y|)\left|\nabla_{y} u(y, z)\right|^{2} d y d z \\
& \geq \int_{\mathbf{R}^{N-k}} \int_{\mathbf{R}^{k}} V(|y|)\left|\frac{y}{|y|} \cdot \nabla_{y} u(y, z)\right|^{2} d y d z \\
& \geq \int_{\mathbf{R}^{N-k}} \beta\left(r^{k-1} V, r^{k-1} W ; \infty\right) \int_{\mathbf{R}^{k}} W(|y|)|u(y, z)|^{2} d y d z \\
& =\beta\left(r^{k-1} V, r^{k-1} W ; \infty\right) \int_{\mathbf{R}^{N-k}} \int_{\mathbf{R}^{k}} W(|y|)|u(y, z)|^{2} d y d z
\end{aligned}
$$

Now, we will show that $\beta\left(r^{k-1} V, r^{k-1} W ; \infty\right)$ is the best constant using the idea in [35]. By choosing $u(y, z)=f(y) g(z)$, we have

$$
\int_{\mathbf{R}^{N-k}} \int_{\mathbf{R}^{k}} W(|y|)|u(y, z)|^{2} d y d z=\int_{\mathbf{R}^{k}} W(|y|)|f(y)|^{2} d y \int_{\mathbf{R}^{N-k}}|g(z)|^{2} d z
$$

and

$$
\begin{aligned}
& \int_{\mathbf{R}^{N-k}} \int_{\mathbf{R}^{k}} V(|y|)|\nabla u(y, z)|^{2} d y d z \\
& \leq \int_{\mathbf{R}^{N-k}} \int_{\mathbf{R}^{k}} V(|y|)[|\nabla f(y)||g(z)|+|f(y)||\nabla g(z)|]^{2} d y d z \\
& \leq(1+\varepsilon)^{2} \int_{\mathbf{R}^{N-k}} \int_{\mathbf{R}^{k}} V(|y|)|\nabla f(y)|^{2}|g(z)|^{2} d y d z \\
& \quad+C(\varepsilon) \int_{\mathbf{R}^{N-k}} \int_{\mathbf{R}^{k}} V(|y|)|f(y)|^{2}|\nabla g(z)|^{2} d y d z .
\end{aligned}
$$


Hence,

$$
\begin{aligned}
& \frac{\int_{\mathbf{R}^{N-k}} \int_{\mathbf{R}^{k}} V(|y|)|\nabla u(y, z)|^{2} d y d z}{\int_{\mathbf{R}^{N-k}} \int_{\mathbf{R}^{k}} W(|y|)|u(y, z)|^{2} d y d z} \\
& \leq(1+\varepsilon)^{2} \frac{\int_{\mathbf{R}^{k}} V(|y|)|\nabla f(y)|^{2} d y}{\int_{\mathbf{R}^{k}} W(|y|)|f(y)|^{2} d y}+C(\varepsilon) \frac{\int_{\mathbf{R}^{k}} V(|y|)|f(y)|^{2} d y}{\int_{\mathbf{R}^{k}} W(|y|)|f(y)|^{2} d y} \frac{\int_{\mathbf{R}^{N-k}}|\nabla g(z)|^{2} d z}{\int_{\mathbf{R}^{N-k}}|g(z)|^{2} d z} .
\end{aligned}
$$

By noting that

$$
\inf _{g \in C_{0}^{\infty}\left(\mathbf{R}^{N-k}\right)} \frac{\int_{\mathbf{R}^{N-k}}|\nabla g(z)|^{2} d z}{\int_{\mathbf{R}^{N-k}}|g(z)|^{2} d z}=0,
$$

and letting $\varepsilon \downarrow 0$, we obtain

$$
\begin{aligned}
\inf \frac{\int_{\mathbf{R}^{N-k}} \int_{\mathbf{R}^{k}} V(|y|)|\nabla u(y, z)|^{2} d y d z}{\int_{\mathbf{R}^{N-k}} \int_{\mathbf{R}^{k}} W(|y|)|u(y, z)|^{2} d y d z} & \leq \inf \frac{\int_{\mathbf{R}^{k}} V(|y|)|\nabla f(y)|^{2} d y}{\int_{\mathbf{R}^{k}} W(|y|)|f(y)|^{2} d y} \\
& =\beta\left(r^{k-1} V, r^{k-1} W ; \infty\right) .
\end{aligned}
$$

Now, assume that we have

$$
\int_{\mathbf{R}^{N-k}} \int_{\mathbf{R}^{k}} V(|y|)|\nabla u(y, z)|^{2} d y d z \geq c \int_{\mathbf{R}^{N-k}} \int_{\mathbf{R}^{k}} W(|y|)|u(y, z)|^{2} d y d z
$$

for all $u \in C_{0}^{\infty}\left(B_{R}\right)$ for some $c>0$. For any $f \in C_{0}^{\infty}\left(\mathbf{R}^{k}\right)$ and $g \in C_{0}^{\infty}\left(\mathbf{R}^{N-k}\right)$, we set

$$
u(y, z)=f(y) g(z) .
$$

As above, from

$$
\int_{\mathbf{R}^{N-k}} \int_{\mathbf{R}^{k}} V(|y|)\left|\nabla_{y} u(y, z)\right|^{2} d y d z \geq c \int_{\mathbf{R}^{N-k}} \int_{\mathbf{R}^{k}} W(|y|)|u(y, z)|^{2} d y d z
$$

we get

$$
\begin{aligned}
& (1+\varepsilon)^{2} \frac{\int_{\mathbf{R}^{k}} V(|y|)|\nabla f(y)|^{2} d y}{\int_{\mathbf{R}^{k}} W(|y|)|f(y)|^{2} d y}+C(\varepsilon) \frac{\int_{\mathbf{R}^{k}} V(|y|)|f(y)|^{2} d y}{\int_{\mathbf{R}^{k}} W(|y|)|f(y)|^{2} d y} \frac{\int_{\mathbf{R}^{N-k}}|\nabla g(z)|^{2} d z}{\int_{\mathbf{R}^{N-k}}|g(z)|^{2} d z} \\
& \geq \frac{\int_{\mathbf{R}^{N-k}} \int_{\mathbf{R}^{k}} V(|y|)|\nabla u(y, z)|^{2} d y d z}{\int_{\mathbf{R}^{N-k}} \int_{\mathbf{R}^{k}} W(|y|)|u(y, z)|^{2} d y d z} \geq c
\end{aligned}
$$

By noting again that

$$
\inf _{g \in C_{0}^{\infty}\left(\mathbf{R}^{N-k}\right)} \frac{\int_{\mathbf{R}^{N-k}}|\nabla g(z)|^{2} d z}{\int_{\mathbf{R}^{N-k}}|g(z)|^{2} d z}=0,
$$


and letting $\varepsilon \downarrow 0$, we get

$$
\int_{\mathbf{R}^{k}} V(|y|)|\nabla f(y)|^{2} d y \geq c \int_{\mathbf{R}^{k}} W(|y|)|f(y)|^{2} d y
$$

for any $f \in C_{0}^{\infty}\left(\mathbf{R}^{k}\right)$. Hence, by Theorem $\mathrm{A},\left(r^{k-1} V, r^{k-1} W\right)$ is a 1-dimensional Bessel pair on $(0, \infty)$.

Proof of Theorem 1.2. It is enough to prove that $\int_{\mathbf{R}^{k}} V(|y|)\left|\frac{y}{|y|} \cdot \nabla f(y)\right|^{p} d y \geq$ $\int_{\mathbf{R}^{k}} W(|y|)|f(y)|^{p} d y$ for all $f \in C_{0}^{\infty}\left(\mathbf{R}^{k}\right)$. We first assume that $f$ is radial. In this case, we need to show that

$$
\int_{0}^{\infty}\left(r^{k-1} V(r)\right)\left|f_{r}\right|^{p} d r \geq \int_{0}^{\infty}\left(r^{k-1} W(r)\right)|f|^{p} d r .
$$

We set

$$
g(r)=\frac{f(r)}{\phi(r)}
$$

Then

$$
f_{r}(r)=g_{r}(r) \phi(r)+\phi_{r}(r) g(r) .
$$

By an elementary inequality, we get

$$
\begin{aligned}
\left|f_{r}(r)\right|^{p} & =\left|g_{r}(r) \phi(r)+\phi_{r}(r) g(r)\right|^{p} \\
& \geq\left|\phi_{r}(r)\right|^{p}|g(r)|^{p}+\phi\left|\phi_{r}(r)\right|^{p-2} \phi_{r}(r)\left(|g(r)|^{p}\right)_{r} .
\end{aligned}
$$

Hence

$$
\begin{aligned}
& \int_{0}^{\infty} r^{k-1} V(r)\left|f_{r}\right|^{p} d r \\
& \geq \int_{0}^{\infty} r^{k-1} V(r)\left|\phi_{r}(r)\right|^{p}|g(r)|^{p}+\int_{0}^{\infty} r^{k-1} V(r) \phi\left|\phi_{r}(r)\right|^{p-2} \phi_{r}(r)\left(|g(r)|^{p}\right)_{r} \\
& =\int_{0}^{\infty} r^{k-1} V(r)\left|\phi_{r}(r)\right|^{p}|g(r)|^{p}-\int_{0}^{\infty}\left(r^{k-1} V(r) \phi\left|\phi_{r}(r)\right|^{p-2} \phi_{r}(r)\right)_{r}|g(r)|^{p} \\
& =-\int_{0}^{\infty}\left(r^{k-1} V(r)\left|\phi_{r}(r)\right|^{p-2} \phi_{r}(r)\right)_{r} \phi|g(r)|^{p} .
\end{aligned}
$$

We note that by assumptions we have

$$
-\int_{0}^{\infty}\left(r^{k-1} V(r)\left|\phi_{r}(r)\right|^{p-2} \phi_{r}(r)\right)_{r} \phi|g(r)|^{p} \geq \int_{0}^{\infty} r^{k-1} W(r) \phi^{p-1} \phi|g(r)|^{p} .
$$

Thus

$$
\int_{0}^{\infty} r^{k-1} V(r)\left|f_{r}\right|^{p} d r \geq \int_{0}^{\infty} r^{k-1} W(r)|f|^{p} d r
$$

In the general case, we set

$$
F(r)=\left(\frac{1}{\int_{\mathbf{S}^{k-1}} d \sigma} \int_{\mathbf{S}^{k-1}}|f(r \sigma)|^{p} d \sigma\right)^{\frac{1}{p}} .
$$


By Hölder's inequality, we can deduce that

$$
\left|F_{r}(r)\right| \leq\left(\frac{1}{\int_{\mathbf{S}^{k-1}} d \sigma} \int_{\mathbf{S}^{k-1}}\left|f_{r}(r \sigma)\right|^{p} d \sigma\right)^{\frac{1}{p}} .
$$

Now, we note that

$$
\begin{aligned}
\int_{\mathbf{R}^{k}} W(|y|)|F|^{p} d y & =\left(\int_{\mathbf{S}^{k-1}} d \sigma\right) \int_{0}^{\infty} r^{k-1} W(r)|F|^{p} d r \\
& =\left(\int_{\mathbf{S}^{k-1}} d \sigma\right) \int_{0}^{\infty} r^{k-1} W(r) \frac{1}{\int_{\mathbf{S}^{k-1}} d \sigma} \int_{\mathbf{S}^{k-1}}|f(r \sigma)|^{p} d \sigma d r \\
& =\int_{0}^{\infty} \int_{\mathbf{S}^{k-1}} W(r)|f(r \sigma)|^{p} r^{k-1} d \sigma d r \\
& =\int_{\mathbf{R}^{k}} W(|y|)|f|^{p} d x .
\end{aligned}
$$

Also

$$
\begin{aligned}
\int_{\mathbf{R}^{k}} V(|y|)\left|\frac{y}{|y|} \cdot \nabla F\right|^{p} d y & =\left(\int_{\mathbf{S}^{k-1}} d \sigma\right) \int_{0}^{\infty} r^{k-1} V(r)\left|F_{r}\right|^{p} d r \\
& \leq\left.\left.\left(\int_{\mathbf{S}^{k-1}} d \sigma\right) \int_{0}^{\infty} r^{k-1} V(r)\left|\frac{1}{\int_{\mathbf{S}^{k-1}} d \sigma} \int_{\mathbf{S}^{k-1}}\right| f_{r}(r \sigma)\right|^{p} d \sigma\right|^{p} d r \\
& =\int_{0}^{\infty} \int_{\mathbf{S}^{k-1}} V(r)\left|f_{r}(r \sigma)\right|^{p} r^{k-1} d \sigma d r \\
& =\int_{\mathbf{R}^{k}} V(|y|)\left|\frac{y}{|y|} \cdot \nabla f\right|^{p} d y .
\end{aligned}
$$

Since $F$ is radial, we deduce

$$
\begin{aligned}
\int_{\mathbf{R}^{k}} V(|y|)\left|\frac{y}{|y|} \cdot \nabla f\right|^{p} d y & \geq \int_{\mathbf{R}^{k}} V(|y|)\left|\frac{y}{|y|} \cdot \nabla F\right|^{p} d x \\
& \geq \int_{\mathbf{R}^{k}} W(|y|)|F|^{p} d x=\int_{\mathbf{R}^{k}} W(|y|)|f|^{p} d x .
\end{aligned}
$$

\section{Proof of Theorem 1.3}

Let $u \in C_{0}^{\infty}(\Omega)$. We then extend $u$ as zero outside $\Omega$ and may consider that $u \in C_{0}^{\infty}\left(\mathbf{R}^{N}\right)$. Hence, we can decompose $u$ into spherical harmonics as follows:

$$
u=\sum_{k=0}^{\infty} u_{k}=\sum_{k=0}^{\infty} f_{k}(r) \phi_{k}(\sigma),
$$

where $\phi_{k}(\sigma)$ are the orthonormal eigenfunctions of the Laplace-Beltrami operator with corresponding eigenvalues $c_{k}=k(N+k-2), k \geq 0$. We note that the corresponding components $f_{k}$ are in $C_{0}^{\infty}(\Omega)$ and satisfy $f_{k}(r)=O\left(r^{k}\right), f_{k}^{\prime}(r)=O\left(r^{k-1}\right)$ 
as $r \downarrow 0$. In particular,

$$
\phi_{0}(\sigma)=1, \quad c_{0}=0 \quad \text { and } \quad f_{0}(r)=\frac{1}{\left|\partial B_{r}\right|} \int_{\partial B_{r}} u d s .
$$

We have the following result:

Lemma 3.1. Assume that the decomposition of $u$ into the spherical harmonics is $u=\sum_{k=0}^{\infty} u_{k}=\sum_{k=0}^{\infty} f_{k}(r) \phi_{k}(\sigma)$ and assume that $V$ is a positive radial $C^{1}$-function on $\mathbf{R}^{N} \backslash\{0\}$. Then we have

$$
\begin{aligned}
\int_{\mathbf{R}^{N}} V(|x|)|u|^{2} d x & =\sum_{k=0}^{\infty} \int_{\mathbf{R}^{N}} V(|x|)\left|f_{k}(|x|)\right|^{2} d x, \\
\int_{\mathbf{R}^{N}} V(|x|)|\mathcal{R} u|^{2} d x & =\sum_{k=0}^{\infty} \int_{\mathbf{R}^{N}} V(|x|)\left|\nabla f_{k}(|x|)\right|^{2} d x, \\
\int_{\mathbf{R}^{N}} V(|x|)\left|\mathcal{R}^{2} u+\frac{N-1}{|x|} \mathcal{R} u\right|^{2} d x & =\sum_{k=0}^{\infty} \int_{\mathbf{R}^{N}} V(|x|)\left|\Delta f_{k}(|x|)\right|^{2} d x .
\end{aligned}
$$

Proof. By polar coordinate and direct computations, we get

$$
\begin{aligned}
\int_{\mathbf{R}^{N}} V(|x|)|u|^{2} d x & =\int_{0}^{\infty} \int_{\mathbf{S}^{N-1}} V(r)\left|\sum_{k=0}^{\infty} f_{k}(r) \phi_{k}(\sigma)\right|^{2} r^{N-1} d r d \sigma \\
& =\sum_{k=0}^{\infty} \int_{\mathbf{R}^{N}} V(|x|)\left|f_{k}(|x|)\right|^{2} d x .
\end{aligned}
$$

Also

$$
\begin{aligned}
\int_{\mathbf{R}^{N}} V(|x|)|\mathcal{R} u|^{2} d x & =\int_{0}^{\infty} \int_{\mathbf{S}^{N-1}} V(r)\left|\sum_{k=0}^{\infty} \frac{\partial u_{k}}{\partial r}\right|^{2} r^{N-1} d r d \sigma \\
& =\int_{0}^{\infty} \int_{\mathbf{S}^{N-1}} V(r)\left|\sum_{k=0}^{\infty} f_{k}^{\prime}(r) \phi_{k}(\sigma)\right|^{2} r^{N-1} d r d \sigma \\
& =\sum_{k=0}^{\infty}\left|\mathbf{S}^{N-1}\right| \int_{0}^{\infty} V(r)\left|f_{k}^{\prime}(r)\right|^{2} r^{N-1} d r \\
& =\sum_{k=0}^{\infty} \int_{\mathbf{R}^{N}} V(|x|)\left|\nabla f_{k}(|x|)\right|^{2} .
\end{aligned}
$$

Finally, we have

$$
\begin{aligned}
& \int_{\mathbf{R}^{N}} V(x)\left|\mathcal{R}^{2} u+\frac{N-1}{|x|} \mathcal{R} u\right|^{2} d x \\
& =\int_{0}^{\infty} \int_{\mathbf{S}^{N-1}} V(r)\left|\sum_{k=0}^{\infty}\left(f_{k}^{\prime \prime}(r)+\frac{N-1}{r} f_{k}^{\prime}(r)\right) \phi_{k}(\sigma)\right|^{2} r^{N-1} d r d \sigma \\
& =\sum_{k=0}^{\infty}\left|\mathbf{S}^{N-1}\right| \int_{0}^{\infty} V(r)\left|\left(f_{k}^{\prime \prime}(r)+\frac{N-1}{r} f_{k}^{\prime}(r)\right)\right|^{2} r^{N-1} d r d \sigma \\
& =\sum_{k=0}^{\infty} \int_{\mathbf{R}^{N}} V(x)\left|\Delta f_{k}(|x|)\right|^{2} d x
\end{aligned}
$$


We are now ready to prove Theorem 1.3:

Proof of Theorem 1.3. First, assume that $(V, W)$ is a $N$-dimensional Bessel pair on $(0, R)$. Using Theorem $\mathrm{C}$, we have

$$
\begin{aligned}
\int_{B_{R}} V(x)\left|\Delta f_{k}(|x|)\right|^{2} d x \geq & \beta(V, W ; R) \int_{B_{R}} W(x)\left|\nabla f_{k}(|x|)\right|^{2} d x \\
& +(N-1) \int_{B_{R}}\left(\frac{V(x)}{|x|^{2}}-\frac{V_{r}(x)}{|x|}\right)\left|\nabla f_{k}(|x|)\right|^{2} d x .
\end{aligned}
$$

As a consequence

$$
\begin{aligned}
\sum_{k=0}^{\infty} \int_{B_{R}} V(x)\left|\Delta f_{k}(|x|)\right|^{2} d x \geq & \beta(V, W ; R) \sum_{k=0}^{\infty} \int_{B_{R}} W(x)\left|\nabla f_{k}(|x|)\right|^{2} d x \\
& +(N-1) \sum_{k=0}^{\infty} \int_{B_{R}}\left(\frac{V(x)}{|x|^{2}}-\frac{V_{r}(x)}{|x|}\right)\left|\nabla f_{k}(|x|)\right|^{2} d x .
\end{aligned}
$$

By Lemma 3.1, we obtain

$$
\begin{aligned}
\int_{\mathbf{R}^{N}} V(x)\left|\mathcal{R}^{2} u+\frac{N-1}{|x|} \mathcal{R} u\right|^{2} d x \geq & \beta(V, W ; R) \int_{\mathbf{R}^{N}} W(x)|\mathcal{R} u|^{2} d x \\
& +(N-1) \int_{\mathbf{R}^{N}}\left(\frac{V(x)}{|x|^{2}}-\frac{V_{r}(x)}{|x|}\right)|\mathcal{R} u|^{2} d x .
\end{aligned}
$$

Now, assume that

$$
\begin{aligned}
\int_{\mathbf{R}^{N}} V(x)\left|\mathcal{R}^{2} u+\frac{N-1}{|x|} \mathcal{R} u\right|^{2} d x \geq & c \int_{\mathbf{R}^{N}} W(x)|\mathcal{R} u|^{2} d x \\
& +(N-1) \int_{\mathbf{R}^{N}}\left(\frac{V(x)}{|x|^{2}}-\frac{V_{r}(x)}{|x|}\right)|\mathcal{R} u|^{2} d x
\end{aligned}
$$

for some $c>0$. Then, for any radial function $u \in C_{0}^{\infty}\left(B_{R}\right)$, we get

$$
\int_{B_{R}} V(x)|\Delta u|^{2} d x \geq c \int_{B_{R}} W(x)|\nabla u|^{2} d x+(N-1) \int_{B_{R}}\left(\frac{V(x)}{|x|^{2}}-\frac{V_{r}(x)}{|x|}\right)|\nabla u|^{2} d x .
$$

Hence $(V, W)$ is a $N$-dimensional Bessel pair on $(0, R)$ by Theorem $\mathrm{C}$.

\section{References}

[1] Adimurthi, N. Chaudhuri, and M. Ramaswamy: An improved Hardy-Sobolev inequality and its application. - Proc. Amer. Math. Soc. 130:2, 2002, 489-505.

[2] Adimurthi, M. Grossi, and S. Santra: Optimal Hardy-Rellich inequalities, maximum principle andrelated eigenvalue problem. - J. Funct. Anal. 240, 2006, 36-83.

[3] Badiale, M., and G. Tarantello: A Sobolev-Hardy inequality with applications to a nonlinear elliptic equation arising in astrophysics. - Arch. Ration. Mech. Anal. 163:4, 2002, 259-293.

[4] Balinsky, A. A., W. D. Evans, and R. T. Lewis: The analysis and geometry of Hardy's inequality. - Universitext, Springer, 2015.

[5] Barbatis, G., and A. Tertikas: On a class of Rellich inequalities. - J. Comp. Appl. Math. 194, 2006, 156-172.

[6] Beckner, W.: Multilinear embedding and Hardy's inequality. - In: Some topics in harmonic analysis and applications, Adv. Lect. Math. (ALM) 34, Int. Press, Somerville, MA, 2016, 1-26. 
[7] Berchio, E., D. Cassani, and F. Gazzola: Hardy-Rellich inequalities with boundary remainder terms and applications. - Manuscripta Math. 131, 2010, 427-458.

[8] Brezis, H., and J. L. VÁzquez: Blow-up solutions of some nonlinear elliptic problems. - Rev. Mat. Univ. Complut. Madrid 10:2, 1997, 443-469.

[9] Cianchi, A., and A. Ferone: Hardy inequalities with non-standard remainder terms. - Ann. Inst. H. Poincaré Anal. Non Linéaire 25:5, 2008, 889-906.

[10] Davies, E. B.: A review of Hardy inequalities. - In: The Maz'ya anniversary collection, Vol. 2 (Rostock, 1998), Oper. Theory Adv. Appl. 110, Birkhäuser, Basel, 1999, 55-67.

[11] Edmunds, D. E., and W. D. Evans: The Rellich inequality. - Rev. Mat. Complut. 29, 2016, $511-530$.

[12] W. D. Evans, and R. T. Lewis: On the Rellich inequality with magnetic potentials. - Math. Z. 251, 2005, 267-284.

[13] Filippas, S., and A. Tertikas: Optimizing improved Hardy inequalities. - J. Funct. Anal. 192:1, 2002, 186-233.

[14] Gazzola, F., H.-C. Grunau, and E. Mitidieri: Hardy inequalities with optimal constants and remainder terms. - Trans. Amer. Math. Soc. 356:6, 2004, 2149-2168.

[15] Gesztesy, F., and L. Littlejohn: Factorizations and Hardy-Rellich type inequalities. arXiv:1701.08929.

[16] Ghoussoub, N., and A. Moradifam: Bessel pairs and optimal Hardy and Hardy-Rellich inequalities. - Math. Ann. 349:1, 2011, 1-57.

[17] Ghoussoub, N., and A. Moradifam: Functional inequalities: new perspectives and new applications. - Math. Surveys Monogr. 187, Amer. Math. Soc., Providence, RI, 2013.

[18] Ghoussoub, N., and A. Moradifam: On the best possible remaining term in the Hardy inequality. - Proc. Natl. Acad. Sci. USA 105:37, 2008, 13746-13751.

[19] Goldstein, J., I. Kombe, and A. Yener: A unified approach to weighted Hardy type inequalities on Carnot groups. - Discrete Contin. Dyn. Syst. (to appear).

[20] IokU, N., and M. Ishiwata: A scale invariant form of a critical Hardy inequality. - Int. Math. Res. Not. IMRN 2015:18, 2015, 8830-8846.

[21] Ioku, N., M. Ishiwata, and T. OzawA: Sharp remainder of a critical Hardy inequality. Arch. Math. (Basel) 106:1, 2016, 65-71.

[22] Jin, Y., and S. Shen: Weighted Hardy and Rellich inequality on Carnot groups. - Arch. Math. (Basel) 96:3, 2011, 263-271.

[23] Kufner, A., L. Maligranda, and L.-E. Persson: The Hardy inequality. About its history and some related results. - Vydavatelský Servis, Pilsen, 2007.

[24] Kufner, A., and L.-E. Persson: Weighted inequalities of Hardy type. - World Scientific Publishing Co., Inc., River Edge, NJ, 2003.

[25] LAm, N.: A note on the Hardy inequalities and Caffarelli-Kohn-Nirenberg inequalities. Preprint, 2017.

[26] LAm, N., and G. Lu: Improved Hardy-Rellich and Caffarelli-Kohn-Nirenberg inequalities on some classes of functions. - Preprint, 2017.

[27] Machihara, S., T. Ozawa, and H. Wadade: Scaling invariant Hardy inequalities of multiple logarithmic type on the whole space. - J. Inequal. Appl. 2015:281, 2015, 1-13.

[28] E. Mitidieri: A simple approach to Hardy's inequalities. - Mat. Zametki 67, 2000, 563-572; English transl. in Math. Notes 67, 2000, 479-486.

[29] Moradifam, A.: Optimal weighted Hardy-Rellich inequalities on $H^{2} \cap H_{0}^{1}$. - J. Lond. Math. Soc. (2) 85, 2012, 22-40.

[30] Musina, R.: Optimal Rellich-Sobolev constants and their extremals. - Differential Integral Equations 27, 2014, 579-600. 
[31] OpIC, B., and A. Kufner: Hardy-type inequalities. - Pitman Research Notes in Mathematics Series 219. Longman Scientific \& Technical, Harlow, 1990.

[32] Ruzhansky, M., and D. Suragan: On horizontal Hardy, Rellich, Caffarelli-Kohn-Nirenberg and $p$-sub-Laplacian inequalities on stratified groups. - J. Differential Equations 262:3, 2017, $1799-1821$.

[33] Ruzhansky, M., and D. Suragan: Uncertainty relations on nilpotent Lie groups. - Proc. R. Soc. A 473:20170082, 2017.

[34] Ruzhansky, M., and D. Suragan: Hardy and Rellich inequalities, identities, and sharp remainders on homogeneous groups. - arXiv:1603.06239.

[35] Secchi, S., D. Smets, and M. Willem: Remarks on a Hardy-Sobolev inequality. - C. R. Math. Acad. Sci. Paris 336:10, 2003, 811-815.

[36] Takahashi, F.: A simple proof of Hardy's inequality in a limiting case. - Arch. Math. (Basel) 104:1, 2015, 77-82.

[37] Tertikas, A., and N. B. Zographopoulos: Best constants in the Hardy-Rellich inequalities and related improvements. - Adv. Math. 209:2, 2007, 407-459.

Received 6 March 2017 • Accepted 18 July 2017 